\section{A SIMPLE SPECTROSCOPE AND ITS TEACHINGS.}

\section{I.}

SPECTRUM analysis is now becoming so far-reaching, especially in inquiries having to do with the conditions of the various celestial bodies, that there are many who are anxious to know something of its teachings. To some of these, however, the terms used by men of science, a very necessary shorthand, are unfamiliar and appear hard to understand, because the opportunity of seeing the things they are intended to define, and which they generally do define in most admirable fashion, has never presented itself. I propose, therefore, to attempt to show that there is nothing recondite about these terms; that it is possible without any expensive apparatus for every one, who will take a little trouble, to observe the phenomena for himself, after which the meanings of the terms employed will present no difficulty whatever.

One key to the hieroglyphics, the light story, which is hidden in every ray of light, is supplied to us by the rainbow. It teaches us that the white light with which nature bountifully supplies us in the sun's rays, is composed of rays of different kinds or of different colours ; and it is common knowledge that there is an almost perfect analogy between these coloured lights and sounds of different pitches.

The blue of the rainbow may be likened to the higher notes of the key-board of a piano, and the red of the rainbow, on the other hand, may be likened to the longer sound waves, which produce the lower notes; and as we are able in the language of music to define each particular note, such as B flat and G sharp, and so on, so light-waves are defined by their colours or wave-lengths.

What nature accomplishes by a rain-drop, we can do with a prism or a grating. A prism is a piece of glass or other transparent material through which the light is bent out of its course or refracted in the process. A grating is a collection of wires, or scratches on glass or metal ; equidistant, very near together and all parallel. When light passes through or is reflected by such a system it is said to be diffracted, and one result that we are concerned in, is very similar to that of passing light through a prism.

It is rapidly becoming a familiar fact to many that when a ray of white light is refracted by a prism, or diffracted by a grating, a band of colour similar to a rainbow is produced, and that this effect follows because white light is built up of lights of every colour, each colour having its own special length of wave and degree of refrangibility. Our rainbow band is called a spectrum.

Such a glass prism or grating is the fundamental part of the instrument called the spectroscope, and the most complicated spectroscope which we can imagine, simply utilises the part which the prism or grating plays in breaking up a beam of white light into its constituent parts from the red to the violet. Between these colours we get that string of orange, yellow, green and blue, which we are familiar with in the rainbow.

For sixpence any of us may make for ourselves an instrument which will serve many of the purposes of demonstrating some of the marvellously fertile fields of knowledge which have been recently opened up to us. From an optician we can get a small prism for $6 d$; get a piece of wood from 20 to 10 inches long (the distance of distinct vision), $\mathrm{I}$ inch broad and $\frac{1}{2}$ an inch thick. On one end glue a cork 2 inches high; at the other end fasten, by melting the bottom, a stump of a wax candle of such a height that the dark cone above the wick is level with the top of the cork. Then glue the prism on the cork, so that by looking sideways through the prism the coloured image, or spectrum, of the flame of the candle placed at the other end of the piece of wood can be seen.
We get a band of colour, a spectrum of the candle flame, built up of an infinite number of images of the flame produced by the light rays of every colour. But, so far, the spectrum is impure because the images overlap. We can get rid of this defect by replacing the candle by a needle.

If we now allow the needle to reflect the light of the candle flame, taking care that the direct light from the candle does not fall upon the face of the prism, we then get a much purer band of colour, because now we have an innumerable multitude of images of the thin needle, instead of the broad flame, close together. The needle is the equivalent of the slit of the more complicated spectroscopes used in laboratories.

We can vary this experiment by gumming two pieces of tin-foil with two perfectly straight edges on a piece of glass so that the straight edges are parallel and very near together. In this way we have a slit; this should be fixed close to the candle and between it and the prism.

Now the light of the candle is white, and the preceding experiment tells us that such light gives us a band containing all the colours without any breaks or gaps. We have what is called a continuous spectrum.

\section{The Continuous Spectrum.}

If we burn a piece of paper, or a match, or ordinary coalgas, we get a white light identical to that given us by the candle; solids which do not liquefy when made whitehot, and liquids which do not volatilise under the same 
light; the white condition comes from the gradual addition of blue as the temperature increases.

One of the laws formulated by Kirchhoff in the infancy of spectroscopic inquiry has to do with the kind of radiation given out by bodies at different temperatures. The law affirms that the hotter a mass of matter is the further its spectrum extends into the ultra-violet.

Gaslight is redder than the light of an incandescent lamp because the latter is hotter. The carbons in a socalled arc-lamp give out a bluish-white light because they are hotter still.

By similar reasoning from experiment we are bound to consider the bluish-white stars, the white stars, the yellow, red and blood-red stars to indicate a decreasing order of temperature. ${ }^{1}$

We shall not go far wrong in supposing that the star with the most intense continuous radiation in the ultraviolet is the hottest, independently of absorbing conditions, which, in the absence of evidence to the contrary, we must assume to follow the same law in all.

An inquiry into the facts placed at our disposal by stellar photographs, shows that there is a considerable variation in the distance to which the radiation extends in the ultra-violet, and that the stars can be arranged in order of temperature on this basis.

Judged by this criterion alone, some of the hottest stars so far observed are $\gamma$ Orionis, $\zeta$ Orionis, $a$ Virginis, $\gamma$ Pegasi, $\eta$ Ursæ Majoris, and $\lambda$ Tauri. Of stars of lower, but not much lower, temperature than the above, may be named Rigel, $\zeta$ 'Tauri, $a$ Andromedæ, $\beta$ Persei, $\boldsymbol{a}$ Pegasi, and $\boldsymbol{\beta}$ Tauri.

In this way spectrum analysis helps us with regard to temperatures, both on the earth and in the heavens.

\section{Discontinuous Spectra with Bright Lines.}

Let us next pass from a solid which retains its incandescence like platinum wire without melting, or a liquid which retains its incandescence without volatilising, like molten iron, and see what happens. We have found that when the light entering the slit consists of every colour and every tone, we have a continuous band of colour. If there be any defect in the light we must have a dis. continuous one, for the reason that an image of the slit cannot be produced in any particular part of the spectrum if there be no light of that particular colour to produce it when we deal with coloured flames or vapours or gases rendered incandescent by electricity.

There are many artificial flames which are coloured, and if their light be analysed in the same way as the light of the candle, a perfectly new set of phenomena present themselves.

Let us again make use of our improvised spectroscope, and allow the needle to be illuminated by the flame of a spirit lamp into which salt is gradually allowed to fall ; we see at once why the flame is orange-coloured. It contains no red, yellow, green, blue, or violet rays, so that we should not represent the spectrum by

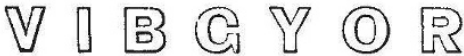

as in the case of the candle, but simply by

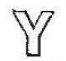

We see one image of the needle coloured in orange. We have passed from the spectrum of polychromatic

1 On this point I wrote as follows in 1892 : "An erroneous idea with regard to the indications of the temperature of the stars has been held hy those who have not considered the matter specially. It has been imagined that the presence of the series of hydrogen lines in the ultra-violet was of itself sufficient evidence of a very high temperature. The experiments of Cornu, however, have shown that the complete series of lines can be seen with an ordinary spark without jar. Hence the high temperature of such a star as Sirius is not indicated by the fact that its spectrum shows the whole series of hydrogen lines, but by the fact that there is bright continuous radiation far in the ultra-violet."

NO. 1529 , VOL. 59] to that of monochromatic light-from white light to coloured light-from light of all wave-lengths to light of one wave-length; from an infinite number of slit images giving a continuous band of every colour, to one image of the slit produced by light of one refrangibility, the colour of the image depending upon the refrangibility. What we shall see in passing from the spectrum of the candle to that of sodium vapour in the spirit lamp is shown in the accompanying woodcut.

That we are truly dealing with an image of the needle (or a slit) can be proved by using a slit of any shape. This can be shown by slightly altering our needle ex-

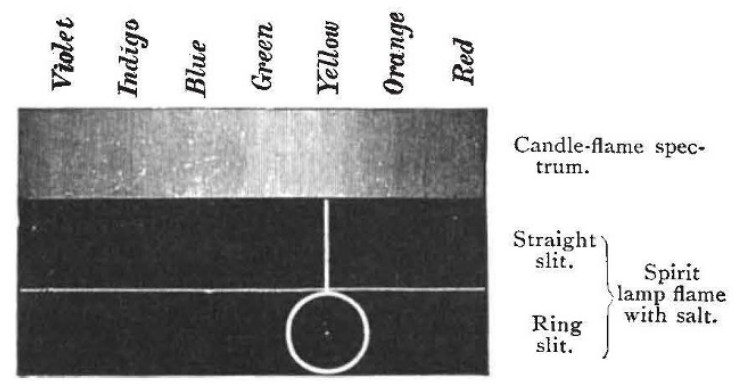

Fig. 2.-A continuous and a discontinuous spectrum.

periment. Take a piece of glass and a piece of tin-foil I $\frac{1}{2}$ inches square, cut out of the centre of the tin-foil a disc slightly larger than a threepenny-piece, and gum the remainder on the glass. In the centre, where the disc has been cut away, gum a threepenny-piece. The interval between the threepenny-piece and the tin-foil constitutes a circular slit. Let it replace the needle, and examine the flame of the spirit lamp charged with salt through it with the prism as before.

It will readily be grasped, from what has been stated, that in the case of coloured flames, the light passing through the spectroscope being only red, or yellow, or

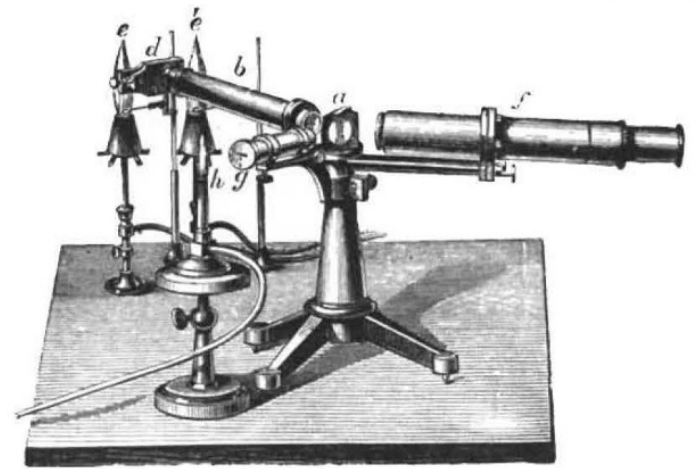

FIG. 3.-Observation of flame spectrum with ordinary spectroscope with comparison prism. $a$, prism; $b$, collimator; $d$, slit ; ée, flames to be compared ; $f$, observing telescope; $g$, scale illuminated by $h$ and reflected by the second surface of the prism into the telescope.

green, as the case may be, will go to build up an image of the slit in the appropriate part of the spectrum, and that the image thus built up will take the form of a line or circle, according to the slit we use.

Many chemical substances, salts or various metals, become luminous by inserting them into flames, as we have treated common salt (chloride of sodium). With each metal the colour imparted to the flame is different. The resulting spectrum is called a discontinuous spectrum, because it is only here and there that images of the slit are produced; because some coloured rays, and not all, are present. 
The usual laboratory arrangement for observing the spectra of flames is shown in the woodcuts.

Further, the system of images of the needle (or slit)

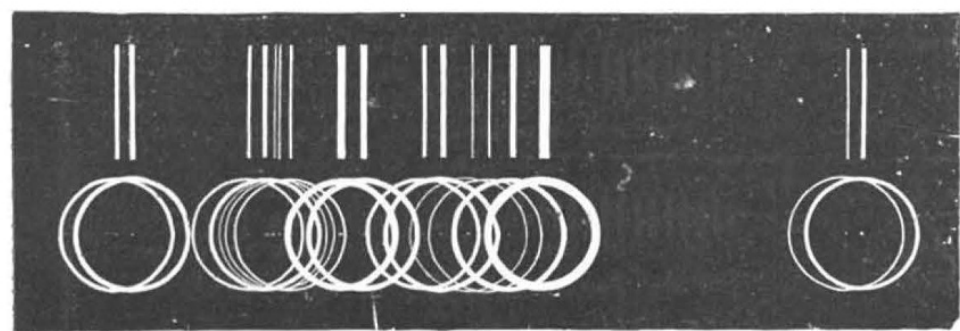

FIG. 4.-The spectrum of a complicated light-source as seen with a circular and a line slit.

inch. The unit of wave-length usually employed is the ten-millionth of a millimetre. These wave-lengths get shorter as we pass from the red to the violet.

So much then in general for the radiations given out by light sources, and the manner in which the spectroscope shows them, and the student records their positions.

Spectrum analysis was established when experiment proved that no two substances which give a line spectrum give the same order of lines from one end of the spectrum to the other; in other words, the line spectrum of each chemical substance differs from that given by any other.

Here then is one of the secrets of the new power of investigation of which the

varies for each substance, and it is on this ground that the term spectrum analysis is used, because we can in this way recognise the various substances in the flame.

But we are not limited to flame temperatures; substances in a state of gas or vapour may be made to glow by electricity. At these higher temperatures very complicated spectra are produced, and again the spectrum is special to each chemical substance experimented on; the iniages of the needle (or slit), occupying different positions along the spectrum according to the nature of the source of light.

Fig. 3 gives us a laboratory prism spectroscope of small dispersion; with the more complicated spectra the phenomena are often better seen if more than one prism are employed. Fig. 5 shows an instrument in which four prisms are used.

For accurate measures of the wave-lengths of the lines a grating is employed as shown in Fig 6.

It is in the case of the more complicated spectra that the wave-length has to be specially considered from the point of view of defining the position of a line. It is not enough to say, as was said in the case of the sodium line, that it is located in the orange.

The lengths of the various light-waves are very small. The wave-length of the sound-wave of the middle $C$ of a piano is about 4 feet, while the wave-length of yellow light as defined by that of a line very accurately measured

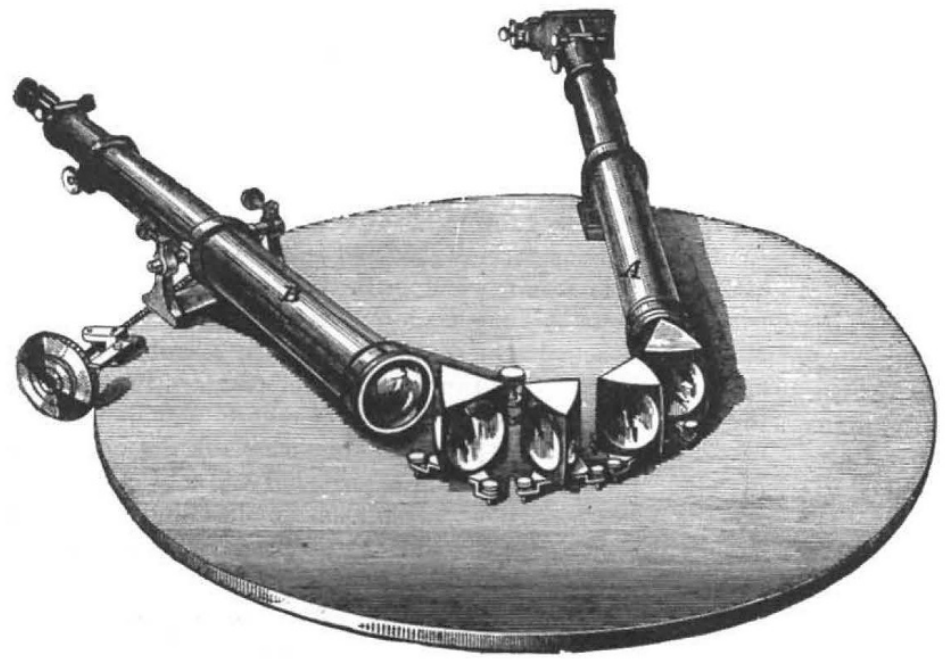

F1G. 5.-Steinheil spectroscope with four prisms.

is 0005895 of a millimetre, that is 5895 ten-millionths of a millimetre; so that there are 43,130 waves in a British thoroughly typical. pectroscope has put us in possession: we can recognise each element by ts spectrum, whether that spectrum is

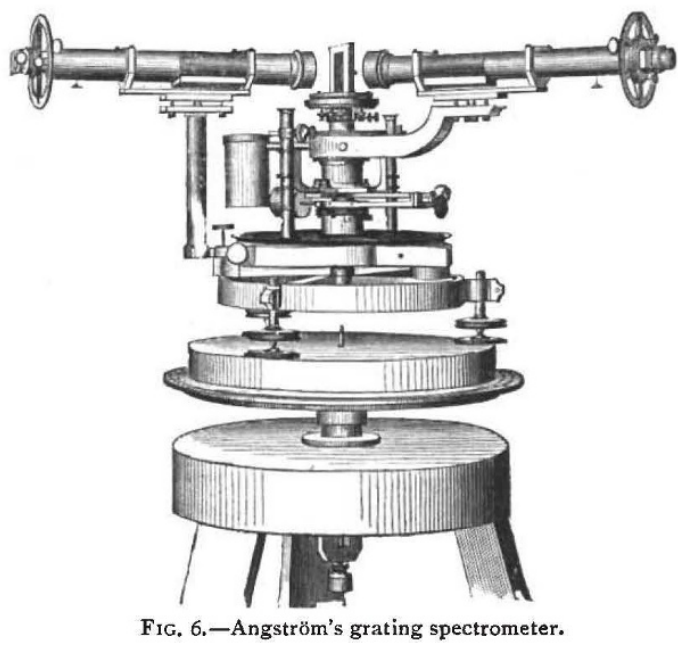

produced in the laboratory or is given by light travelling earthwards from the most distant star, provided the element exists both here and there.

It is in this way that spectrum analysis helps us with regard to chemistry; the spectrum varies according to the chemical substance which produces it.

\section{Flutings.}

The earliest spectroscopic observations revealed the fact that in some spectra the lines, instead of being irregularly distributed along the spectrum, were arranged in an easily seen rhythmic fashion. Such allocations of lines are called flutings, as a succession of them gives rise to an appearance strongly recalling the flutings of a Corinthian column seen under a strong side light.

Our improvised spectroscope helps us here too; use the candle and straight slit in front of it as before, but shorten the slit, and only allow the blue light from the base of the candle flame to pass through it to the prism. We see two or three sets of flutings. These are the flutings of carbon, and they are amongst the most beautiful examples known and are ( To be continued.) NORMAN LOCKYER. NO. I 529, vOL. 59] 